\title{
Cell Population Data NE-SFL and MO-WX From Sysmex XN-3000 Can Provide Additional Information for Exclusion of Acute Promyelocytic Leukemia From Other Acute Myeloid Leukemias: A Preliminary Study
}

Sang Hyuk Park, M.D..$^{1,2}$, Hyung-Hoi Kim, M.D. ${ }^{1,2}$, In-Suk Kim, M.D. ${ }^{3}$, Jongyoun Yi, M.D. ${ }^{1,2}$, Chulhun L. Chang, M.D. ${ }^{3}$, and Eun Yup Lee, M.D.,

Department of Laboratory Medicine ${ }^{1}$, Pusan National University School of Medicine, Pusan National University Hospital, Busan; Biomedical Research Institute ${ }^{2}$, Pusan National University Hospital, Busan; Department of Laboratory Medicine ${ }^{3}$, Pusan National University School of Medicine, Pusan National University Yangsan Hospital, Yangsan, Korea

Dear Editor,

Acute promyelocytic leukemia (APL) is diagnosed by detection of PML-RARA using reverse transcriptase (RT)-PCR, but reporting of results takes at least two days, and this situation is an obstacle for prompt management of patients. Treatment and disease course in APL are different from those in other AMLs because APL patients require all-trans-retinoic acid (ATRA) for remission and for reducing the risk of disseminated intravascular coagulation (DIC), which is a dangerous complication of APL. Hence, treatment of APL with ATRA can be more important than starting chemotherapy in other AMLs, and rapid exclusion of APL from other AMLs is important, allowing for rapid implementation of appropriate treatment. Nonetheless, discrimination of APL from other AMLs by morphological evaluation can cause misdiagnosis in some cases with APL-like morphology. The re- cently launched Sysmex XN-3000 analyzer (Sysmex, Kobe, Japan) can show various cell population data (CPD), and some of these characteristics, e.g., NE-SFL [fluorescent light intensity of the neutrophil area on the WDF (white blood cell differential) scattergram] and NE-WY (fluorescent light distribution width of the neutrophil area on the WDF scattergram) provide useful information for detection of sepsis [1]. We evaluated the performance of CPD items in peripheral blood (PB) for differential diagnosis of APL and other AMLs.

Ten APL patients and 21 patients with other AMLs diagnosed from July 2014 to December 2015 at Pusan National University Hospital, Korea were prospectively enrolled. Thirty-one PB samples obtained at diagnosis were analyzed on an XN-3000 analyzer. Demographic characteristics, complete blood cell counts (CBC) results, and $46 \mathrm{CPD}$ items were compared between APL
Received: April 27, 2016

Revision received: June 15, 2016

Accepted: July 20, 2016

\footnotetext{
Corresponding author: Sang Hyuk Park

Department of Laboratory Medicine, Pusan National University School of Medicine, Pusan National University Hospital, 179 Gudeok-ro, Seo-gu, Busan 49241, Korea

Tel: +82-51-240-7463, Fax: +82-51-247-6560

E-mail: korailman-1@hanmail.net
}

Co-corresponding author: Hyung-Hoi Kim

Department of Laboratory Medicine, Pusan National University School of Medicine, Pusan National University Hospital, 179 Gudeok-ro, Seo-gu, Busan 49241, Korea

Tel: +82-51-240-7414, Fax: +82-51-247-6560, E-mail: hhkim@pusan.ac.kr

\section{(C) The Korean Society for Laboratory Medicine.}

This is an Open Access article distributed under the terms of the Creative Commons Attribution Non-Commercial License (http://creativecommons.org/licenses/by-nc/4.0) which permits unrestricted non-commercial use, distribution, and reproduction in any medium, provided the original work is properly cited. 
Table 1. Comparison of the routine and cell population data (CPD) items obtained on a XN-3000 automatic blood cell analyzer between patients with AML and those with acute promyelocytic leukemia (APL)

\begin{tabular}{|c|c|c|c|c|c|c|c|}
\hline \multirow{2}{*}{ Items } & \multicolumn{2}{|c|}{ Results, median (range) } & \multirow{2}{*}{$P$ value } & \multirow{2}{*}{ Items } & \multicolumn{2}{|c|}{ Results, median (range) } & \multirow{2}{*}{$P$ value } \\
\hline & AML $(N=21)$ & APL $(N=10)$ & & & AML $(N=21)$ & APL $(N=10)$ & \\
\hline Sex (M:F) & 12: 9 & $5: 5$ & 0.709 & LY-WX & $501.0(289.0-709.0)$ & $502.5(421.0-782.0)$ & 0.833 \\
\hline Age (yr) & $65.0(19.0-83.0)$ & $43.0(26.0-68.0)$ & 0.021 & LY-WY & $915.0(713.0-1,750.0)$ & $767.5(615.0-1,017.0)$ & 0.008 \\
\hline WBC $\left(\times 10^{9} / L\right)$ & $8.94(0.45-204.0)$ & $3.57(0.44-59.86)$ & 0.043 & LY-WZ & $604.0(503.0-845.0)$ & $590.0(517.0-840.0)$ & 0.800 \\
\hline $\mathrm{HGB}(\times 10 \mathrm{~g} / \mathrm{L})$ & $8.8(6.6-11.7)$ & $9.9(7.8-11.6)$ & 0.019 & MO-WX & $351.0(67.0-514.0)$ & $237.0(56.0-375.0)$ & 0.009 \\
\hline $\mathrm{PLT}\left(\times 10^{9} / \mathrm{L}\right)$ & $33.0(8.0-202.0)$ & $41.0(13.0-377.0)$ & 0.447 & MO-WY & $862.0(33.0-1,313.0)$ & $692.5(32.0-904.0)$ & 0.049 \\
\hline PB Blasts (\%) & $30.0(10.0-80.0)$ & $3.0(1.0-20.0)$ & $<0.001$ & MO-WZ & $675.0(271.0-932.0)$ & $713.5(42.0-825.0)$ & 0.899 \\
\hline PB Promyelocytes (\%) & $0.0(0.0-2.0)$ & $72.5(50.0-90.0)$ & $<0.001$ & WBC-N $\left(\times 10^{9} / \mathrm{L}\right)$ & $8.94(0.45-204.0)$ & $3.57(0.44-59.86)$ & 0.053 \\
\hline \multirow{2}{*}{$\begin{array}{l}\text { FAB classification (M1: M2: } \\
\text { M3: M4: M5: M7) }\end{array}$} & \multirow[t]{2}{*}{ 3:13:0:1:3:1 } & \multirow[t]{2}{*}{ 0:0:10:0:0:0 } & \multirow[t]{2}{*}{$<0.001$} & WBC-D $\left(\times 10^{9} / L\right)$ & $8.78(0.46-202.98)$ & $3.40(0.41-59.09)$ & 0.053 \\
\hline & & & & TNC-N $\left(\times 10^{9} / \mathrm{L}\right)$ & $8.97(0.46-204.63)$ & $3.59(0.45-60.09)$ & 0.053 \\
\hline $\operatorname{NRBC}\left(\times 10^{12} / \mathrm{L}\right)$ & $0.02(0.00-2.65)$ & $0.02(0.00-0.23)$ & 0.415 & TNC-D $\left(\times 10^{9} / \mathrm{L}\right)$ & $8.81(0.47-203.61)$ & $3.42(0.42-59.32)$ & 0.053 \\
\hline NRBC (\%) & $0.20(0.00-11.40)$ & $0.45(0.00-2.30)$ & 0.468 & Delta NEUT $\left(\times 10^{9} / \mathrm{L}\right)$ & $1.26(0.07-76.97)$ & $1.41(0.08-7.72)$ & 0.512 \\
\hline NEUT $\left(\times 10^{9} / L\right)$ & $2.43(0.08-111.49)$ & $1.43(0.20-7.96)$ & 0.473 & Delta NEUT (\%) & $16.90(1.30-74.30)$ & $39.0(5.40-82.60)$ & 0.118 \\
\hline LYMPH $\left(\times 10^{9} / \mathrm{L}\right)$ & $3.05(0.26-45.67)$ & $0.91(0.16-13.52)$ & 0.056 & Delta LYMPH $\left(\times 10^{9} / \mathrm{L}\right)$ & $3.00(0.26-45.16)$ & $0.91(0.15-13.52)$ & 0.051 \\
\hline MONO $\left(\times 10^{9} / \mathrm{L}\right)$ & $3.16(0.06-108.62)$ & $0.40(0.01-38.33)$ & 0.051 & Delta LYMPH (\%) & $26.90(3.40-91.90)$ & $29.80(12.60-58.50)$ & 0.866 \\
\hline EOSINO $\left(\times 10^{9} / L\right)$ & $0.01(0.00-16.09)$ & $0.01(0.00-0.32)$ & 0.628 & HFLC (×109/L) & $0.03(0.00-0.76)$ & $0.00(0.00-0.01)$ & 0.060 \\
\hline $\operatorname{BASO}\left(\times 10^{9} / \mathrm{L}\right)$ & $0.03(0.00-0.59)$ & $0.01(0.00-0.04)$ & 0.082 & HFLC (\%) & $0.20(0.00-7.60)$ & $0.00(0.00-2.30)$ & 0.267 \\
\hline NEUT (\%) & $21.30(1.30-74.80)$ & $51.75(13.30-83.20)$ & 0.076 & BASO-D $\left(\times 10^{9} / \mathrm{L}\right)$ & $0.04(0.00-1.69)$ & $0.01(0.00-0.06)$ & 0.054 \\
\hline LYMPH (\%) & $30.20(3.40-91.90)$ & $30.40(12.60-58.50)$ & 0.966 & BASO-D (\%) & $0.40(0.00-2.20)$ & $0.05(0.00-2.30)$ & 0.165 \\
\hline MONO (\%) & $27.30(3.90-70.60)$ & $15.20(1.00-64.00)$ & 0.099 & RBC-He (pg) & $31.50(16.90-38.00)$ & $30.05(28.70-38.00)$ & 0.767 \\
\hline EOSINO (\%) & $0.10(0.00-7.90)$ & $0.00(0.00-4.30)$ & 0.856 & Delta-He (pg) & $2.30(-6.90$ to 5.80$)$ & $4.20(-0.20$ to 6.40$)$ & 0.113 \\
\hline BASO (\%) & $0.30(0.00-1.40)$ & $0.15(0.00-0.70)$ & 0.354 & RET-Y (ch) & $179.2(110.7-195.2)$ & $184.6(168.0-195.8)$ & 0.272 \\
\hline $\mathrm{IG}\left(\times 10^{9} / \mathrm{L}\right)$ & $0.05(0.00-34.52)$ & $0.04(0.00-0.24)$ & 0.719 & RET-RBC-Y (ch) & $174.5(112.6-184.8)$ & $169.5(157.7-183.1)$ & 0.499 \\
\hline IG (\%) & $0.70(0.00-16.90)$ & $0.55(0.00-25.00)$ & 0.672 & IRF-Y (ch) & $171.3(145.6-196.6)$ & $181.5(160.4-199.3)$ & 0.183 \\
\hline NE-SSC (ch) & $148.8(124.8-181.6)$ & $141.0(123.7-165.6)$ & 0.375 & $\mathrm{RPI}$ & $0.40(0.10-2.40)$ & $0.45(0.00-2.40)$ & 0.881 \\
\hline NE-SFL (ch) & $52.2(42.9-89.7)$ & $45.5(40.7-52.4)$ & 0.004 & RET-UPP & $2.00(0.00-81.00)$ & $4.00(0.00-101.00)$ & 0.913 \\
\hline NE-FSC (ch) & $74.8(44.0-91.9)$ & $74.0(58.6-90.6)$ & 0.673 & RET_TNC & $105.0(4.0-1,883.0)$ & $25.5(9.0-470.0)$ & 0.070 \\
\hline LY-X (ch) & $84.8(74.3-97.7)$ & $83.3(78.5-117.9)$ & 0.342 & HYPO-He (\%) & $0.70(0.10-47.90)$ & $0.55(0.20-3.70)$ & 0.983 \\
\hline LY-Y (ch) & $73.6(62.9-99.6)$ & $76.6(64.3-108.9)$ & 0.099 & HYPER-He (\%) & $0.40(0.10-3.10)$ & $0.40(0.20-0.90)$ & 0.651 \\
\hline LY-Z (ch) & $61.3(54.6-65.6)$ & $63.5(59.8-70.7)$ & 0.056 & $\operatorname{FRC}\left(\times 10^{12} / L\right)$ & $0.014(0.000-0.180)$ & $0.011(0.000-0.090)$ & 0.526 \\
\hline MO-X (ch) & $120.7(101.2-141.0)$ & $123.2(107.0-130.6)$ & 0.460 & FRC (\%) & $0.64(0.00-5.07)$ & $0.41(0.01-2.67)$ & 0.597 \\
\hline MO-Y (ch) & $119.3(82.8-223.8)$ & $113.5(56.5-142.0)$ & 0.291 & $\operatorname{IPF}\left(\times 10^{9} / \mathrm{L}\right)$ & $2.90(0.10-15.50)$ & $1.20(0.50-13.90)$ & 0.190 \\
\hline MO-Z (ch) & $65.4(57.5-76.8)$ & $68.6(48.0-77.6)$ & 0.486 & H-IPF & $1.60(0.30-34.10)$ & $0.90(0.20-9.10)$ & 0.197 \\
\hline NE-WX & $439.0(33.0-649.0)$ & $362.0(278.0-574.0)$ & 0.499 & MicroR (\%) & $2.50(0.40-48.10)$ & $2.90(0.70-5.90)$ & 0.597 \\
\hline NE-WY & $998.0(97.0-3,086.0)$ & $780.0(537.0-2,295.0)$ & 0.139 & MacroR (\%) & $5.40(2.50-21.10)$ & $3.45(2.60-10.40)$ & 0.066 \\
\hline NE-WZ & $900.0(91.0-1,285.0)$ & $852.5(685.0-1,382.0)$ & 0.999 & & & & \\
\hline
\end{tabular}

$P$ values were obtained by the Mann-Whitney $U$ test (for all items except sex and FAB classification) and $\chi^{2}$ test (for sex and $F A B$ classification).

Abbreviations and definitions: WBC, white blood cells; HGB, hemoglobin; PLT, platelets; PB peripheral blood; FAB, French-America-British; NRBC, nucleated red blood cells; NEUT, neutrophils; LYMPH, lymphocytes; MONO, monocytes; EOSINO, eosinophils; BASO, basophils; IG, immature granulocytes; NESSC, the lateral scattered light intensity of the NEUT area on the WDF scattergram; NE-SFL, the fluorescent light intensity of the NEUT area on the WDF scattergram; NE-FSC, the forward-scattered light intensity of the NEUT area on the WDF scattergram; LY-X, the lateral scattered light intensity of the LYMPH area on the WDF scattergram; LY-Y, the fluorescent light intensity of the LYMPH area on the WDF scattergram; LY-Z, the forward-scattered light intensity of the LYMPH area on the WDF scattergram; MO-X, the lateral scattered light intensity of the MONO area on the WDF scattergram; MO-Y, the fluorescent light intensity of the MONO area on the WDF scattergram; MO-Z, the forward-scattered light intensity of the MONO area on the WDF scattergram; NE-WX, the lat- 
Table 1. Continued

eral scattered light distribution width of the NEUT area on the WDF scattergram; NE-WY, the fluorescent light distribution width of the NEUT area on the WDF scattergram; NE-WZ, the forward-scattered light distribution width of the NEUT area on the WDF scattergram; LY-WX, the lateral scattered light distribution width of the LYMPH area on the WDF scattergram; LY-WY, the fluorescent light distribution width of the LYMPH area on the WDF scattergram; LY-WZ, the forward-scattered light distribution width of the LYMPH area on the WDF scattergram; MO-WX, the lateral scattered light distribution width of the MONO area on the WDF scattergram; MO-WY, the fluorescent light distribution width of the MONO area on the WDF scattergram; MO-WZ, the forward-scattered light distribution width of the MONO area on the WDF scattergram; WBC-N, the WBC count calculated from the WNR channel; WBC-D, the WBC count calculated from the WDF channel; TNC-N, the total nucleated cell count (WBC+NRBC) calculated from the WNR channel; TNC-D, the total nuclear cell count (WBC+NRBC) calculated from the WDF channel; Delta NEUT, the number of particles obtained by subtracting the IG count from the NEUT count; Delta LYMPH, the number of particles obtained by subtracting the HFLC count from the LYMPH count; HFLC, the count of the upper LYMPH area of the WDF scattergram; BASO-D, the basophil counts calculated from the WDF channel; RBC-He, the correlation between RBC-Y and MCH to convert RBC-Y into [pg] units; Delta-He, subtraction of RBC-He from RET-Hb; RET-Y, the forward scattered light intensity of the RET area on the RET scattergram; RET-RBC-Y, the forward scattered light intensity of RBC area on the RET scattergram; IRF-Y, the intensity of forward scattered light from the IRF area on the RET scattergram; RPI, reticulocyte production index; RET-UPP, the count in the upper area of the RET scattergram; RET-TNC, the count in the TNC area of the RET scattergram; HYPO-He, the ratio of the count in the low level area of the forward scattered light signal in the RBC (mature red blood cell) area of the RET scattergram, to mature red blood cells; HYPER-He, the ratio of the count in the high-level area of the forward scattered light signal in the RBC (mature red blood cell) area of the RET scattergram to mature red blood cells; FRC, the count in a specific area below the RBC area in the RET scattergram; IPF, immature platelet fraction; $\mathrm{H}-\mathrm{IPF}$, the ratio to the total platelet count of the count of platelets that appear in the area of stronger fluorescent light intensity within the IPF area of the PLT-F scattergram; MicroR, Micro RBC ratio; MacroR, Macro RBC ratio; ch, channel.

Table 2. ROC analysis results on the four cell population data (CPD) items with significant differences for discrimination of patients with acute promyelocytic leukemia from those with other AMLs

\begin{tabular}{|c|c|c|c|c|c|c|c|c|c|c|c|}
\hline \multirow{2}{*}{ Items } & \multirow{2}{*}{$\begin{array}{c}\text { AUC } \\
(95 \% \mathrm{Cl})\end{array}$} & \multirow{2}{*}{$\begin{array}{l}\text { Best } \\
\text { cutoff }\end{array}$} & \multirow{2}{*}{$\begin{array}{l}\text { Sensitivity } \\
(95 \% \text { Cl) }\end{array}$} & \multirow{2}{*}{$\begin{array}{l}\text { Specificity } \\
(95 \% \text { CI) }\end{array}$} & \multirow{2}{*}{ NPV } & \multirow{2}{*}{ PPV } & \multirow{2}{*}{$\begin{array}{l}\text { Accuracy } \\
(95 \% \text { Cl) }\end{array}$} & \multicolumn{4}{|c|}{$P$ value ${ }^{*}$} \\
\hline & & & & & & & & NE-SFL & LY-WY & MO-WX & MO-WY \\
\hline NE-SFL & $\begin{array}{c}0.829 \\
(0.651-0.939)\end{array}$ & $\leq 49.8$ & $\begin{array}{c}90.0 \\
(69.6-98.2)\end{array}$ & $\begin{array}{c}66.7 \\
(45.4-82.8)\end{array}$ & 93.3 & 56.3 & $\begin{array}{c}74.2 \\
(56.8-86.3)\end{array}$ & NA & 0.772 & 0.715 & 0.335 \\
\hline LY-WY & $\begin{array}{c}0.800 \\
(0.618-0.921)\end{array}$ & $\leq 808.0$ & $\begin{array}{c}70.0 \\
(39.7-89.2)\end{array}$ & $\begin{array}{c}81.0 \\
(60.0-92.3)\end{array}$ & 85.0 & 63.6 & $\begin{array}{c}77.4 \\
(60.2-88.6)\end{array}$ & 0.772 & NA & 0.963 & 0.405 \\
\hline MO-WX & $\begin{array}{c}0.795 \\
(0.612-0.918)\end{array}$ & $\leq 260.0$ & $\begin{array}{c}70.0 \\
(39.7-89.2)\end{array}$ & $\begin{array}{c}90.5 \\
(71.1-97.4)\end{array}$ & 86.4 & 77.8 & $\begin{array}{c}83.9 \\
(67.4-92.9)\end{array}$ & 0.715 & 0.963 & NA & 0.270 \\
\hline MO-WY & $\begin{array}{c}0.721 \\
(0.532-0.866)\end{array}$ & $\leq 774.0$ & $\begin{array}{c}80.0 \\
(49.0-94.3)\end{array}$ & $\begin{array}{c}61.9 \\
(40.9-79.3)\end{array}$ & 86.7 & 50.0 & $\begin{array}{c}67.7 \\
(50.1-81.4)\end{array}$ & 0.335 & 0.405 & 0.270 & NA \\
\hline \multirow{2}{*}{ Combinations } & \multirow{2}{*}{$\begin{array}{c}\text { AUC } \\
(95 \% \mathrm{Cl})\end{array}$} & \multirow{2}{*}{$\begin{array}{l}\text { Best } \\
\text { cutoff }\end{array}$} & \multirow{2}{*}{$\begin{array}{l}\text { Sensitivity } \\
(95 \% \text { Cl) }\end{array}$} & \multirow{2}{*}{$\begin{array}{l}\text { Specificity } \\
(95 \% \mathrm{Cl})\end{array}$} & \multirow{2}{*}{ NPV } & \multirow{2}{*}{ PPV } & \multirow{2}{*}{$\begin{array}{l}\text { Accuracy } \\
(95 \% \text { Cl) }\end{array}$} & \multicolumn{4}{|c|}{$P$ value* } \\
\hline & & & & & & & & NE-SFL & LY-WY & MO-WX & MO-WY \\
\hline $\begin{array}{r}\text { NE-SFL } \leq 49.8 \text { or } \\
\text { MO-WX } \leq 260.0\end{array}$ & $\begin{array}{c}0.786 \\
(0.602-0.911)\end{array}$ & NA & $\begin{array}{c}100.0 \\
(72.3-100.0)\end{array}$ & $\begin{array}{c}57.1 \\
(36.5-75.5)\end{array}$ & 100.0 & 52.6 & $\begin{array}{c}71.0 \\
(53.4-83.9)\end{array}$ & 0.982 & 0.816 & 0.891 & 0.543 \\
\hline $\begin{array}{c}\text { NE-SFL } \leq 49.8 \text { and } \\
M 0-W X \leq 260.0\end{array}$ & $\begin{array}{c}0.800 \\
(0.618-0.921)\end{array}$ & NA & $\begin{array}{c}60.0 \\
(31.3-83.2)\end{array}$ & $\begin{array}{c}100.0 \\
(84.5-100.0)\end{array}$ & 84.0 & 100.0 & $\begin{array}{c}87.1 \\
(71.1-94.9)\end{array}$ & 0.891 & 0.679 & 0.982 & 0.451 \\
\hline
\end{tabular}

${ }^{*} P$ values characterize the results of comparison of AUC values between two items.

Abbreviations: AUC, area under the curve; $\mathrm{Cl}$, confidence interval; NPV, negative predictive value; PPV, positive predictive value; NE-SFL, the fluorescent light intensity of the NEUT area on the WDF scattergram; LY-WY, the fluorescent light distribution width of the LYMPH area on the WDF scattergram; MO-WX, the lateral scattered light distribution width of the MONO area on the WDF scattergram; MO-WY, the fluorescent light distribution width of the MONO area on the WDF scattergram; NA, not applicable.

patients and other AML patients. For CPD items with significant differences, ROC curve analysis was performed to evaluate the performance in differential diagnosis of APL and other AMLs and to determine the best cutoff values for APL. Using these cutoff values, the sensitivity, specificity, negative predictive value (NPV), positive predictive value (PPV), and accuracy for each item were calculated. This study was approved by the institutional review board of Pusan National University Hospital.

Results of APL patients showed significantly lower values on four CPD items [NE-SFL, LY-WY (fluorescent light distribution width of the lymphocyte area on the WDF scattergram), MO-WX (lateral scattered light distribution width of the monocyte area on the WDF scattergram), and MO-WY (fluorescent light distribution width of the monocyte area on the WDF scattergram)] compared with those of other AML patients (Table 1). NE-SFL showed the greatest AUC (0.829) and higher sensitivity (90.0\%) and NPV (93.3\%) than other characteristics did in the discrimination of APL from other AMLs with the cutoff $\leq 49.8$. In con- 
trast, MO-WX showed higher specificity (90.5\%), PPV (77.8\%), and accuracy (83.9\%) with the cutoff $\leq 260.0$. Additional analysis revealed that the combination "NE-SFL $\leq 49.8$ or MO-WX $\leq 260.0$ " shows the highest sensitivity $(100.0 \%)$ and NPV (100.0\%), whereas the combination "NE-SFL $\leq 49.8$ and MOWX $\leq 260.0$ " yields the highest specificity (100.0\%) and PPV (100.0\%) (Table 2).

Low NE-SFL values may reflect increased infiltration of more mature granulocytes (such as promyelocytes) than myeloblasts because low fluorescence intensity indicates a low RNA/DNA ratio in more mature cells [1, 2]. APL involves abundant promyelocytes, and this situation may partly explain our results. Significantly lower MO-WX in APL patients may be the result of lower monocyte counts in APL (versus AML), as found in our study, possibly yielding small distribution width.

Clinically, rapid exclusion of APL from other AMLs may be more important than accurate confirmation of APL by more time-consuming diagnostic procedures. The present study showed that CPD items, NE-SFL and MO-WX, would be useful for the discrimination of APL from other AMLs, especially for rapid exclusion of APL with high sensitivity and NPV. The discriminating power of the PB promyelocyte count may be better, but the time benefit of the two CPD items may be greater. Thus, we hypothesize the use of NE-SFL and MO-WX as criteria for rapid exclusion of $\mathrm{APL}$, not as diagnostic parameters, because we cannot diagnose APL without PML-RARA, even if NE-SFL and MO-WX are lower than cutoffs. The sample size here is small and therefore, our results should be regarded as a preliminary data, and more comprehensive research such as a multicenter study should be performed to confirm our preliminary results.

\section{Authors' Disclosures of Potential Conflicts of Interest}

No potential conflicts of interest relevant to this article were reported.

\section{REFERENCES}

1. Park SH, Park CJ, Lee BR, Nam KS, Kim MJ, Han MY, et al. Sepsis affects most routine and cell population data (CPD) obtained using the Sysmex XN-2000 blood cell analyzer: neutrophil-related CPD NE-SFL and NE-WY provide useful information for detecting sepsis. Int J Lab Hematol 2015;37:190-8.

2. Luo Y, Lin J, Chen H, Zhang J, Peng S, Kuang M. Utility of neut-X, neut$Y$ and neut-Z parameters for rapidly assessing sepsis in tumor patients. Clin Chim Acta 2013;422:5-9. 